\title{
Coronary stent as a tubular flow heater in magnetic resonance imaging
}

Stanislav Vrtnik ${ }^{1,2}$, Magdalena Wencka ${ }^{3}$, Andreja Jelen ${ }^{1,2}$, Hae Jin Kim ${ }^{4}$ and Janez Dolinšek ${ }^{1,2^{*}}$

\begin{abstract}
Background: A coronary stent is an artificial metallic tube, inserted into a blocked coronary artery to keep it open. In magnetic resonance imaging (MRI), a stented person is irradiated by the radio-frequency electromagnetic pulses, which induce eddy currents in the stent that produce Joule (resistive) heating. The stent in the vessel is acting like a tubular flow heater that increases the temperature of the vessel wall and the blood that flows through it, representing a potential hazard for the stented patient.

Methods: Heating of a metallic coronary stent in MRI was studied theoretically and experimentally. An analytical theoretical model of the stent as a tubular flow heater, based on the thermodynamic law of heat conduction, was developed. The model enables to calculate the time-dependent stent's temperature during the MRI examination, the increase of the blood temperature passing through the stent and the distribution of the temperature in the vessel wall surrounding the stent. The model was tested experimentally by performing laboratory magnetic resonance heating experiments on a non-inserted stainless-steel coronary stent in the absence of blood flow through it. The model was then used to predict the temperature increase of the stainless-steel coronary stent embedded in a coronary artery in the presence of blood flow under realistic MRI conditions.
\end{abstract}

Results: The increase of the stent's temperature and the blood temperature were found minute, of the order of several tenths of a degree, because the blood flow efficiently cools the stent due to a much larger heat capacity of the blood as compared to the heat capacity of the stent. However, should the stent in the vessel become partially re-occluded due to the restenosis problem, where the blood flow through the stent is reduced, the stent's temperature may become dangerously high.

Conclusions: In the normal situation of a fully open (unoccluded) stent, the increase of the stent temperature and the blood temperature exiting the stent were found minute, of less than $1{ }^{\circ} \mathrm{C}$, so that the blood flow efficiently cools the stent. However, should the problem of restenosis occur, where the blood flow through the stent is reduced, there is a risk of hazardous heating.

Keywords: Coronary stent; Magnetic resonance imaging; Radiofrequency field heating effect; Modeling biomedical systems; MRI safety

\section{Background}

In medicine, a stent is an artificial "tube" inserted into a natural passage/conduit in the body to prevent, or counteract, a disease-induced, localized flow constriction. The most widely known stent use is in the coronary arteries (Hubner 1998), by employing a bare-metal stent, a drug-eluting stent or occasionally a covered

\footnotetext{
* Correspondence: jani.dolinsek@ijs.si

'Jožef Stefan Institute, Jamova 39, SI-1000 Ljubljana, Slovenia

${ }^{2}$ Faculty of Mathematics and Physics, University of Ljubljana, Jadranska 19,

SI-1000 Ljubljana, Slovenia

Full list of author information is available at the end of the article
}

stent. Coronary stents are inserted during a percutaneous coronary intervention (PCI) procedure to keep the blocked coronary arteries open. Stents are also applied to the urinary tract (Yachia 1998), where ureteral stents are used to ensure patency of a ureter, which may be compromised, for example, by a kidney stone. Prostatic stents (Yachia 1998) are needed if a man is unable to urinate due to an enlarged prostate. Stents are also used in a variety of vessels aside from the coronary arteries and as a component of peripheral artery angioplasty.

\section{实 Springer}

(c) 2015 Vrtnik et al.; licensee Springer. This is an open access article distributed under the terms of the Creative Commons Attribution License (http://creativecommons.org/licenses/by/4.0), which permits unrestricted use, distribution, and reproduction in any medium, provided the original work is properly credited. 
A coronary stent is a tubular metal mesh, attached initially in its collapsed form onto the outside of a balloon catheter. In the angioplasty procedure, the physician threads the stent through the lesion in the vessel and expands the balloon that deforms the stent to its expandable size by matching the undeformed vessel diameter. After removal of the deflated balloon, the framework of the stent remains in direct contact with the vessel wall (Figure 1), where it is overgrown by the endothelial tissue in the course of subsequent months. The coronary stent remains permanently inserted in the vessel, representing a metallic object firmly incorporated into the human body for life.

Stents can be assembled from a range of metallic alloys, including stainless steel, nitinol (nickel-titanium) and cobalt-chromium. The presence of a metallic object in the body brings up several safety issues in Magnetic Resonance Imaging (MRI) diagnostics. The two most important are the influence of magnetic forces on the implanted stent (Ahmed and Shellock 2001; Jost and Kumar 1998; Scott and Pettigrew 1994; Shellock and Shellock 1999; Strohm et al. 1999; Kagetsu and Litt 1991; Woods 2007; Lopič et al. 2013) and the stent heating by the radio-frequency (rf) electromagnetic pulses (Shellock and Morisoli 1994; Nyenhuis et al. 2005; Shellock 2011). In MRI, a stented person is irradiated by the rf pulses, which induce eddy currents in the stent that produce Joule (resistive) heating of the stent and its surroundings. During the MRI, the stent in the vessel is acting much like a tubular flow heater that increases the temperature of the vessel wall and the blood that flows through it. Due to the possible heat-induced protein coagulation and the formation of blood clots, stent heating in MRI deserves careful attention. In this paper, we present an analytical theoretical model of the stent as a tubular flow heater in the MRI examination. The model is based on the thermodynamic law of heat conduction and enables us to calculate the time-dependent stent temperature during the MRI examination, the increase of the blood temperature passing through the stent and the distribution of the temperature in the vessel wall surrounding the stent. We have tested the model experimentally by performing laboratory magnetic resonance $\mathrm{rf}$

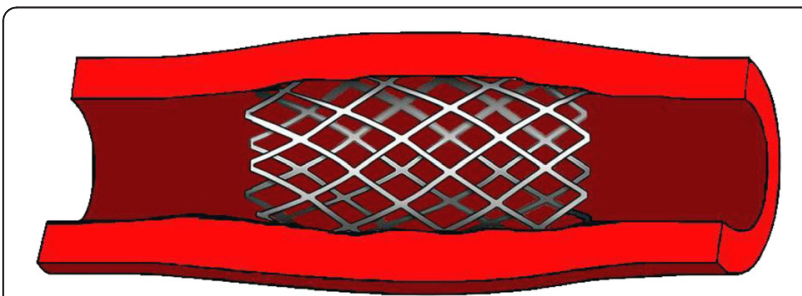

Figure 1 Schematic presentation of the coronary stent in the vessel. heating experiments on a non-inserted stainless-steel coronary stent in the absence of blood flow through it and good matching to the theory was found. The model was then used to predict the temperature increase of the stainless-steel coronary stent embedded in a coronary artery in the presence of blood flow through it under realistic MRI conditions. The results indicate that the increase of the stent's temperature and the blood temperature are minute, of the order of several tenths of a degree, because the blood flow efficiently cools the stent due to a much larger heat capacity of the blood as compared to the heat capacity of the stent. However, should the problem of restenosis occur, where the stent in the vessel becomes partially re-occluded and the flow reduces, there is a risk of hazardous heating and the stent's temperature may become dangerously high.

\section{Methods}

Stent description and characterization

Our experiments were performed on a commercial balloon-expandable coronary stent (HORUSS HDS 1625, International Biomedical Systems, Trieste). The stent was fabricated of a surgical stainless steel, having the nominal length of $16 \mathrm{~mm}$, the nominal diameter of $2.5 \mathrm{~mm}$ and the nominal pressure for the expansion of 8 atm. A small part of the stent was cut away for the physical property determination of the material, whereas the large part (12 mm length) was used for the rf heating experiments. The photograph of the employed stent is shown in Figure 2a.

The stent's material was surgical stainless steel type 316LVM (where "L" stands for Low Carbon and "VM" denotes Vacuum Melted), using a wire of $200-\mu \mathrm{m}$ diameter (Figure 2b). The 316LVM is an austenitic steel, which is widely used for implants because it never develops surface rust and shows superior resistance to constant salt water exposure. According to the international standards (ASM International Handbook Committee 1990), the elemental composition of 316LVM is in the range 0.03 wt.\% $\mathrm{C}$, up to $2.0 \% \mathrm{Mn}, 2.0-3.0 \%$ Mo, up to $1.0 \% \mathrm{Si}, 16.0$ - $18.0 \% \mathrm{Cr}, 10.0-14.0 \% \mathrm{Ni}$, up to $\max .0 .16 \% \mathrm{~N}$, and about $65 \% \mathrm{Fe}$ as the majority element. We have determined the particular chemical composition of our investigated stent by the energydispersive X-ray spectrometer (EDS) using a scanning electron microscope (SEM) Supra VP 35 Zeiss (Carl Zeiss AG, Oberkochen, Germany). The resulting chemical composition (in weight \%) is displayed as a histogram in Figure 2c. The too high carbon concentration $(2.94 \%)$ is artificial; it originates from the contamination of the SEM by the hydrocarbons (a known problem in SEM microscopy). Apart from the carbon, other elements are within the specifications for the 316LVM steel. 

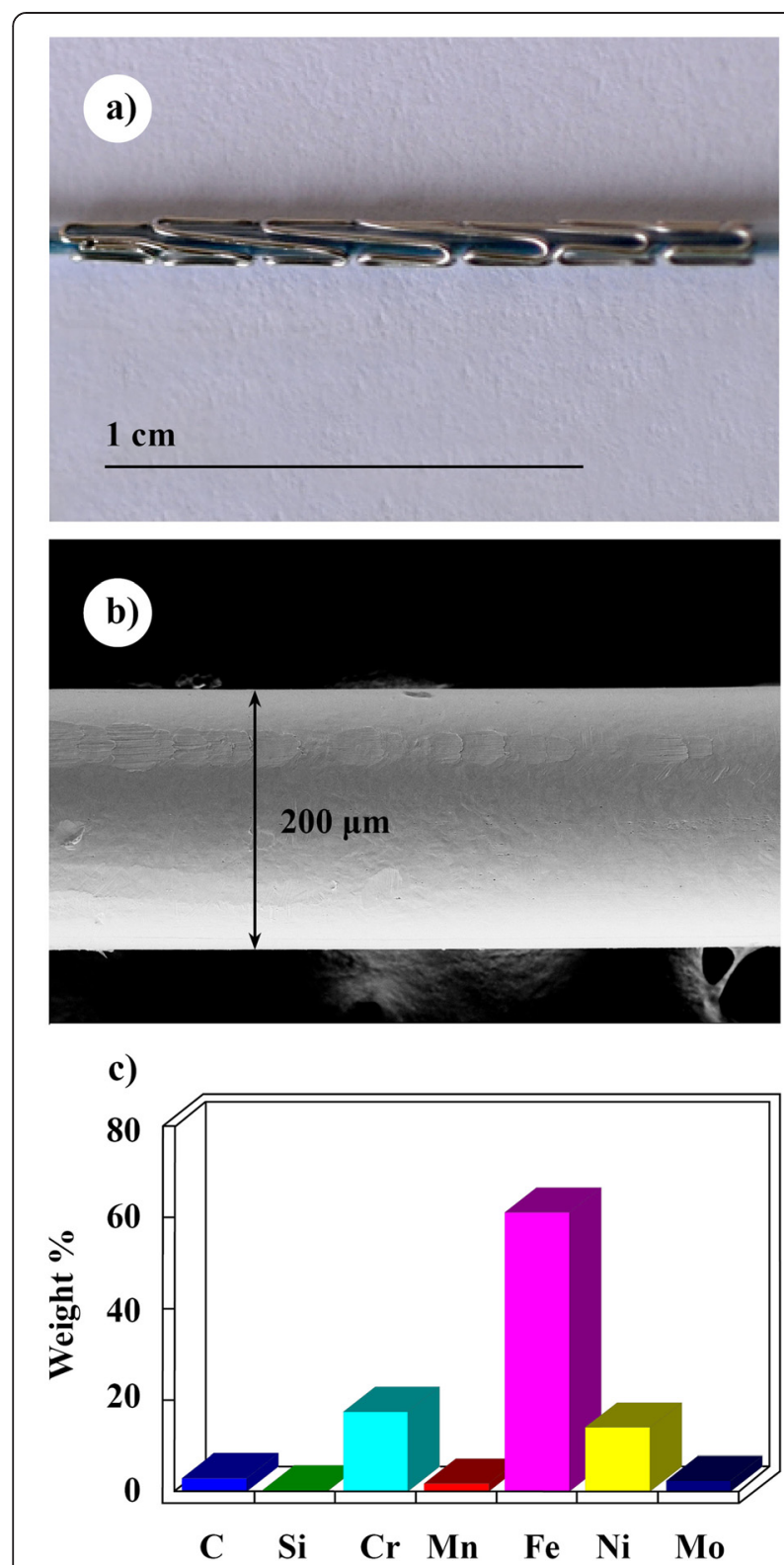

Figure 2 The shape and chemical composition of the employed stent. (a) A photograph of the stent in its collapsed form. (b) A SEM secondary-electron image of the stent's wire of 200- $\mu$ m diameter. (c) Chemical composition (in weight \%) of the stent obtained by EDS analysis, displayed as a histogram.

Since the induction of the eddy currents by the rf field is strong in metallic alloys of low electrical resistivity, we have measured the electrical resistivity $\rho$ of the stent's wire. The direct current $(\mathrm{dc})$ resistivity was determined in the temperature interval from 2 to $360 \mathrm{~K}$ by a standard four-contact method using a Quantum Design PPMS (Physical Property Measurement System). The dc resistivity is usually good approximation to the frequencydependent (ac) resistivity $\rho(\omega)$ up to the microwave frequencies, so that it is considered valid also in the radio-frequency range of the MRI experiments. The result is shown in Figure 3, where it is evident that $\rho(T)$ exhibits a positive temperature coefficient at the values in the range $\rho \approx 100-150 \mu \Omega \mathrm{cm}$, typical of moderately electrically conducting alloys. At the body temperature of $37^{\circ} \mathrm{C}$, the resistivity amounts $\rho_{37^{\circ} \mathrm{C}}=146 \mu \Omega \mathrm{cm}$, which is low enough that a significant induction-heating effect may be expected under the rf-pulse irradiation.

To verify the analytical model of the stent as a tubular flow heater presented in this paper, the specific heat $c_{s}$ of the stent must be known. We have determined $c_{s}$ experimentally by using a Quantum Design PPMS. The graph of the temperature-dependent $c_{s}$ in the interval from 2 to $370 \mathrm{~K}$ is presented in Figure 4, showing that at $37^{\circ} \mathrm{C}, c_{s}=0.46 \mathrm{~J} / \mathrm{gK}$.

\section{Rf heating experiments}

Rf heating experiments of the stent were conducted in a standard $4.7 \quad \mathrm{~T}$ vertical-bore NMR spectrometer operating at the proton resonance frequency $v_{0}\left({ }^{1} \mathrm{H}\right)=$ $200 \mathrm{MHz}$. An AMT $300 \mathrm{~W}$ rf power transmitter was used. To monitor the temperature of the stent, a platinum Pt100 resistor was glued to its outer surface by a thermally conducting varnish (GE/IMI 7031). The stent with the sensor was wrapped up into a Teflon foil of $1.0 \mathrm{~mm}$ total thickness. The output of the sensor was connected to a data logger (PT-104 PT 100 Converter, Pico Technology, Cambridgeshire, UK) that has digitized the signal and enabled us to follow the timedependent increase of the stent's temperature under the $\mathrm{rf}$ irradiation.

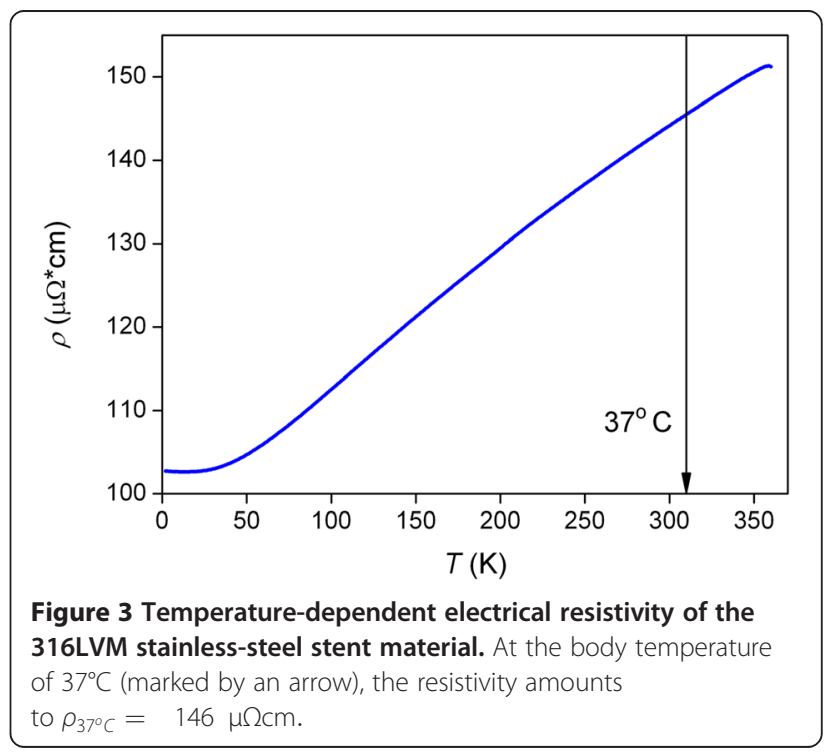




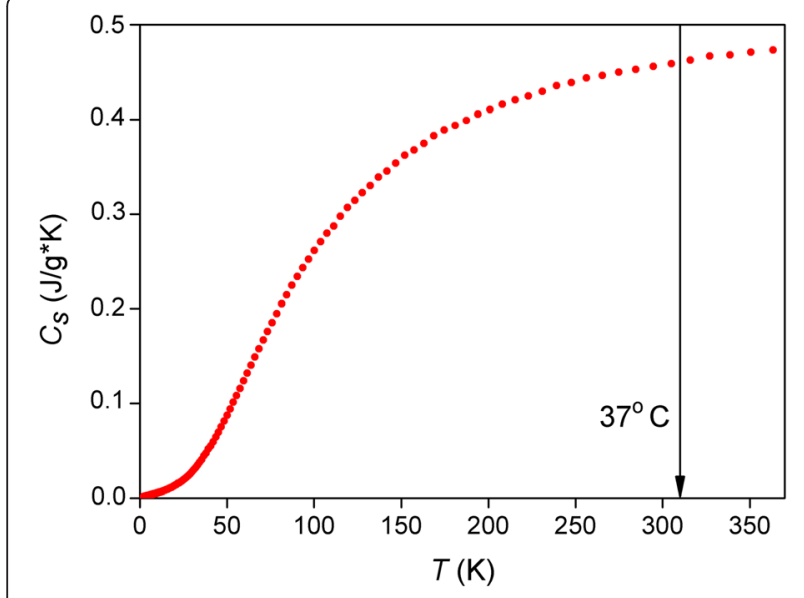

Figure 4 Specific heat $c_{5}$ of the stent in the temperature interval from 2 to $370 \mathrm{~K}$. At the body temperature of $37^{\circ} \mathrm{C}$ (marked by an arrow), $c_{s}=0.46 \mathrm{~J} / \mathrm{gK}$.

\section{Results and discussion}

Analytical model of the stent as a tubular flow heater in MRI We approximate the actual stent's geometry of a cylindrical metal mesh by a homogeneous thin cylinder of the length $l$ and the radius $R$ (Figure 5). The mass of the stent is $m_{s}$ and its specific heat at constant pressure is $c_{s}$. The stent is surrounded by the vessel wall of cylindrical shape with the wall thickness $d$ and the thermal conductivity $\lambda$. The stent's temperature is $T_{s}$, whereas the temperature at the outer surface of the vessel is $T_{0}$ (taken roughly as the body temperature $37^{\circ} \mathrm{C}$ ). The blood mass flow $\Phi_{b}=d m_{b} / d t$ (where $m_{b}$ is the mass of the blood) enters the stent at the body temperature $T_{0}$

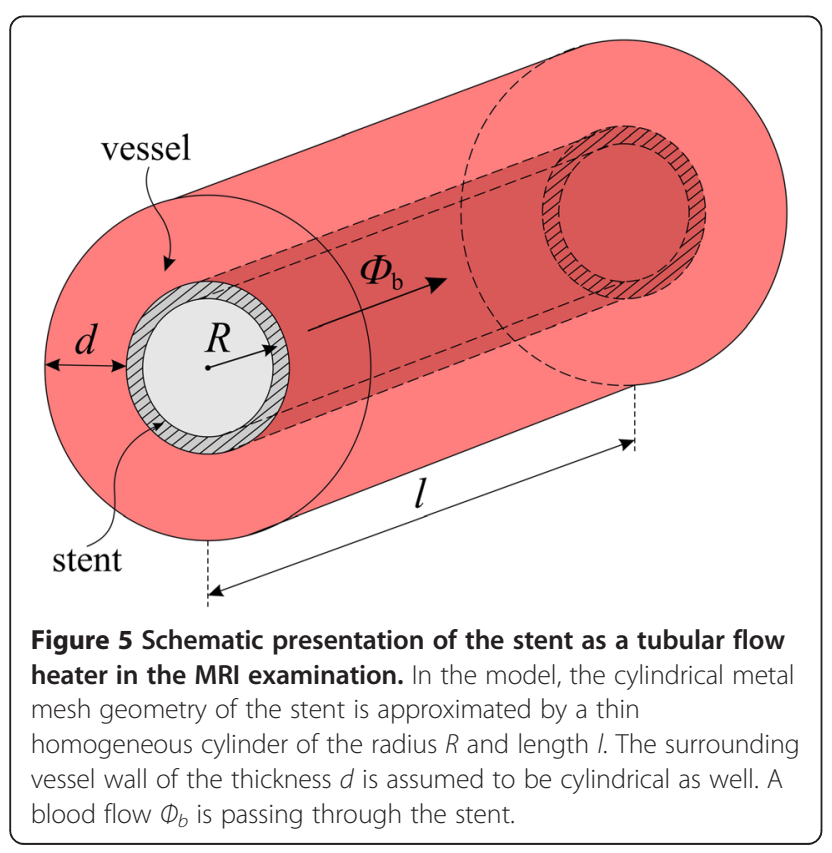

and exits at an elevated temperature $T_{b}$. Due to the heart pulsing, the blood flow is pulsed, but we approximate it by a stationary flow $\Phi_{b}=\rho_{b} S_{s} \bar{v}_{b}$, where $\rho_{b}$ is the blood density, $S_{s}=\pi R^{2}$ is the stent's cross section and $\bar{v}_{b}$ is the average blood velocity through the stent. The specific heat at constant pressure of the blood is $c_{b}$.

The rf electromagnetic pulses during the MRI examination supply the stent with the rf power $P_{r f}$, which transforms into the heat $d Q=P_{r f} d t$ via the Joule heating by the induced eddy currents. A part of this heat is released through the vessel wall. For a cylindrical vessel, the thermal power (heat flow) $P_{v}$ through the wall amounts (Halliday et al. 2005)

$$
P_{v}=\frac{\lambda 2 \pi l}{\ln (1+d / R)}\left(T_{s}-T_{0}\right)
$$

The blood flow through the stent takes away the thermal power

$$
P_{b}=\Phi_{b} c_{b}\left(T_{b}-T_{0}\right)
$$

The heat imbalance $\left(P_{r f}-P_{v}-P_{b}\right) d t$ increases the stent's temperature by $d T_{s}$,

$$
\left(P_{r f}-P_{v}-P_{b}\right) d t=m_{s} c_{s} d T_{s}
$$

Using Equations 1 and 2, we rewrite Equation 3 in the form

$$
\begin{aligned}
P_{r f} & -\frac{\lambda 2 \pi l}{\ln (1+d / R)}\left(T_{s}-T_{0}\right)-\Phi_{b} c_{b}\left(T_{b}-T_{0}\right) \\
& =m_{s} c_{s} \frac{d T_{s}}{d t}
\end{aligned}
$$

which contains two unknown variables, $T_{s}(t)$ and $T_{b}(t)$. To proceed, we assume that the time-dependence of the blood temperature $T_{b}$ follows the time-dependence of the stent's temperature $T_{s}$, though $T_{b}$ may be lower than $T_{s}$,

$$
T_{b}-T_{0}=\varepsilon\left(T_{s}-T_{0}\right),
$$

with $0 \leq \varepsilon \leq 1$. Equation 4 is then cast into the form

$$
\frac{d T_{s}}{d t}+\alpha\left(T_{s}-T_{0}\right)=\frac{P_{r f}}{m_{s} c_{s}},
$$

where

$$
\alpha=\frac{1}{m_{s} c_{s}}\left(\frac{\lambda 2 \pi l}{\ln (1+d / R)}+\varepsilon \Phi_{b} c_{b}\right) .
$$

The solution of Equation 6 is

$$
T_{s}=T_{0}+\Delta T\left(1-e^{-\alpha t}\right),
$$

where $\Delta T=P_{r f} / \alpha m_{s} c_{s}$. The stent reaches the new steadystate temperature $T_{s}(t \rightarrow \infty)=T_{0}+\Delta T$ exponentially with the time constant $\alpha^{-1}$ after the start of the rf pulsing in 
the MRI examination. Using the definition of $\alpha$, the increase of the stent's temperature can also be written in the form

$$
\Delta T=\frac{P_{r f}}{\frac{\lambda 2 \pi l}{\ln (1+d / R)}+\varepsilon \Phi_{b} c_{b}} .
$$

The time-dependent stent's temperature given by Equation 8 is shown in Figure 6a.

According to the above model, the blood temperature $T_{b}(t)$ follows the stent's temperature $T_{s}(t)$, as expressed by Equation 5 . The efficiency of the heat transfer from the stent to the blood is given by the empirical factor $\varepsilon$, which assumes a value between 1 and 0 , depending on the details of the vessel and the blood flow through it. $\varepsilon$ should be determined experimentally for a particular vessel and the type of inserted stent. The timedependent blood temperature obeys the equation $T_{b}(t)$ $=T_{0}+\varepsilon\left(T_{s}(t)-T_{0}\right)$, where $T_{s}(t)$ is given by Equation 8 .
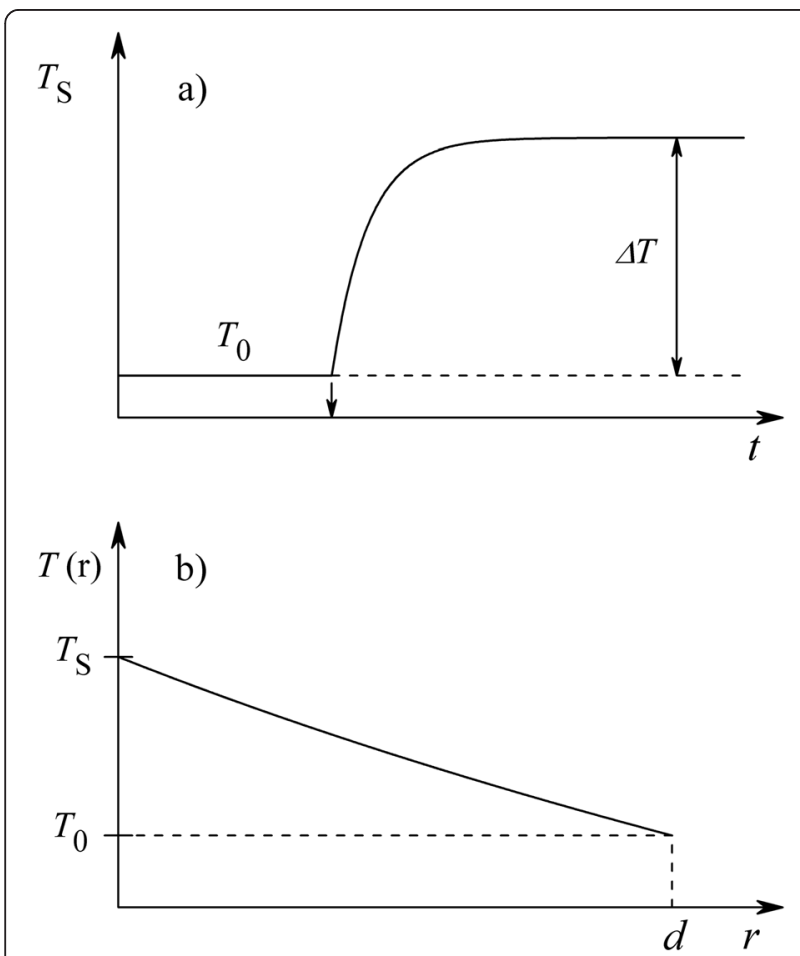

Figure 6 Theoretical stent's temperature in the MRI examination. (a) The time-dependent stent's temperature $T_{s}(t)$ given by Equation 8 . The start of the if irradiation is marked by an arrow on the time axis. The time-dependent blood temperature $T_{b}(t)$ exiting the stent has the same form, except that the steady-state temperature increase is reduced to $\varepsilon \Delta T$ with $0 \leq \varepsilon \leq 1$. (b) Radial distribution of the temperature $T(r)$ within the vessel wall surrounding the stent $(0 \leq r \leq d)$ in the steady state, as given by Equation 11 . At the contact surface to the stent, the vessel wall heats up to the stent temperature, $T(r=0)=T_{s}$, whereas at the vessel's outer surface, the temperature drops to the body temperature, $T(r=d)=T_{0}$, via a $\ln (1+r / R)$ radial dependence.
The $T_{s}(t)$ curve shown in Figure 6a, scaled by a factor $\varepsilon$, is thus valid for the blood temperature $T_{b}(t)$ as well. After the steady-state is reached during the irradiation, the increase of the blood temperature passing the stent is $\varepsilon \Delta T$ with $\Delta T$ given by Equation 9 .

The radial distribution of the temperature $T(r)$ within the vessel wall surrounding the stent $(0 \leq r \leq d)$ in the steady state is obtained by assuming a stationary radial heat flow through the wall, so that $P_{v}$ as given by Equation 1 is constant at any radial distance $r$ from the stent. The condition

$$
P_{v}=\frac{\lambda 2 \pi l\left(T_{s}-T(r)\right)}{\ln (1+r / R)}=\text { const. }
$$

yields the $T(r)$ dependence

$$
T(r)=T_{s}-\frac{P_{v} \ln (1+r / R)}{\lambda 2 \pi l},
$$

which is shown in Figure 6b. At the contact surface to the stent, the vessel wall heats up to the stent temperature, $T(r=0)=T_{s}$, whereas at the vessel's outer surface, the temperature drops to the body temperature, $T(r=d)=T_{0}$, via a $\ln (1+r / R)$ radial dependence.

In the above analytical model of the stent as a tubular flow heater, some simplifications were used mostly to ensure mathematical tractability of the calculation. Two of them deserve to be discussed in more detail. In the model, the vessel wall surrounding the stent is considered to be cylindrical of the thickness $d$, where the temperature at the contact surface to the stent is assumed to equal the stent temperature $T_{\mathrm{s}}$, whereas at the outer surface it equals the body temperature $T_{0}$. The vessel wall thickness was not specified, but for the coronary arteries and the coronary stents it is reasonable to assume the inequality $d<R$, where $R$ is the radius of the stent. This approximation assumes a very powerful cooling process in the body and an excessive temperature gradient within the vessel wall. In fact human temperature regulation is not powerful enough to justify this assumption. In reality, the temperature in the tissue surrounding the stent will drop to the body temperature over the distance of the order of several $R$, thus considerably larger than the vessel wall thickness. In the case where the vessel and the tissue behind it have similar thermal conductivity $\lambda$ values (a reasonable assumption), this discrepancy can be removed by simply taking larger $d$ value in Equations 1 to 9 . Since $d$ always appears in a logarithmic function of the form $\ln (1+d / R)$, which is located in the denominator of the expression for $\Delta T$ (Equation 9), larger $d$ will reduce the heat flow $P_{v}$ through the vessel wall. Due to the logarithmic dependence on $d$, the changes are relatively weak (e.g., for a thickness increase from 
$d=R$ to $d=10 R$, the function $\ln (1+d / R)$ increases by a factor 3.4 only). Smaller $P_{v}$ will increase the stent's steady-state temperature $\Delta T$, thus subjecting the inner side of the vessel to higher temperatures and also heating stronger the blood flowing through the stent. The heating effect is thus increased under the assumption that the temperature of the tissue surrounding the stent drops to the "unperturbed" body temperature at a distance considerably larger than the stent's diameter $R$.

The second simplification is the assumption that the blood temperature follows the stent temperature, as expressed by Equation 5. This assumption has enabled the elimination of one unknown variable (the blood temperature $T_{b}(t)$ ) from the calculation and kept the model simple and analytically tractable. The assumption required the introduction of the empirical "heat-transfer efficiency factor" $\varepsilon$ (with $0 \leq \varepsilon \leq 1$,) describing the efficiency of the heat transfer from the stent to the blood. While it is intuitively plausible to relate the blood temperature to the stent temperature by the Equation 5, the factor $\varepsilon$ is not well-defined as the stent-to-blood heat transfer efficiency will depend on the details of the vessel, the type and geometry of the inserted stent and the blood flow through it. Weaker heat transfer $(\varepsilon \rightarrow 0)$ will increase the risk of high temperatures developed in the stent, as the stent is not giving up the heat to the blood. Good heat transfer can generally be expected for longer stents and lower blood velocity.

\section{Rf heating experiments on a coronary stent in the absence of blood flow}

In our experimental study of the temperature increase of the stainless-steel coronary stent subjected to irradiation by the rf pulses, the stent with the attached Pt100 thermometer was placed into the rf coil of the NMR probe head and inserted into the magnet. A typical MRI rf pulse sequence contains one or more pulses that are usually shaped in the time domain, where a truncated sinc and a Gaussian shape are most commonly employed (Callaghan 1991). Instead of using shaped pulses, our pulse sequence was composed of a train of
50 rectangular pulses of $\tau=20 \mu$ s length each $(1 \mathrm{~ms}$ total pulse duration), separated by $5 \mu$ s and repeated with the repetition rate $t_{0}=100 \mathrm{~ms}$ (Figure 7), yielding the duty cycle $\delta_{D}=0.01$. A shaped rf pulse in a realistic MRI experiment has a similar duration and repetition rate. The power of the rf pulses has been varied during the experiment and the temperature raise of the stent was monitored at different power levels. Here it is worth mentioning that the actual shape of the rf pulses is relatively unimportant for the heating effect; what matters is the average $\mathrm{rf}$ power delivered by the pulses to the stent.

The irradiation of the stent started by switching on the pulse sequence of Figure 7 at a given moment of time and then continuously repeating it at a selected transmitter rf output power level. This resulted in rapid initial growth of the stent temperature, which has saturated to a constant plateau after some time when the balance between the incoming $\mathrm{rf}$ energy and the outgoing heat due to the thermal conduction was achieved. In order to minimize heat losses by thermal conduction to the surrounding air, the rf probe head with the stent was placed into an Oxford continuous-flow cryostat CF 1200 (Oxford Instruments, Abingdon, Oxfordshire, UK), where the air could be evacuated to a pressure down to 0.2 bar. After a steady-state temperature was reached with time at a given $\mathrm{rf}$ power level, the transmitter power was increased and the new stent's temperature was recorded. In the following we present the stent's temperature increase as a function of the average $\mathrm{rf}$ power over the pulse sequence repetition time $t_{0}$, defined as $\bar{P}=\left(1 / t_{0}\right) \int_{0}^{t_{0}} P(t) d t=\delta_{D} P_{t r}$, where $P_{t r}$ is the transmitter power (e.g., $\bar{P}=3 \mathrm{~W}$ for the full transmitter power $P_{t r}=300 \mathrm{~W}$ and the duty cycle $\delta_{D}=$ 0.01). The time-dependent stent's temperature under the rf irradiation at different average power levels $\bar{P}=0.1$, $0.3,1$, and $3 \mathrm{~W}$ is shown in Figure 8a. The initial stent's temperature was $19.5^{\circ} \mathrm{C}$. We observe that at each power level, the rapid initial increase of the temperature slows down with time and reaches a steady state in about 100 s. For the highest average rf power of $\bar{P}=3 \mathrm{~W}$, the stent has reached an astonishingly high temperature

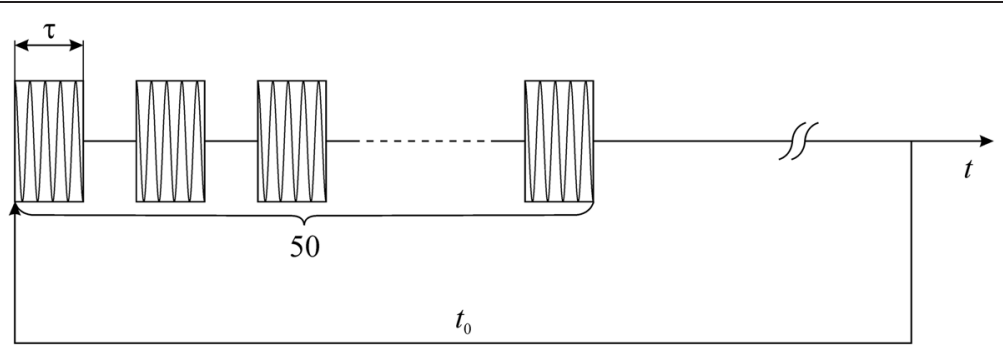

Figure 7 The rf pulse sequence used in the stent heating experiment. A train of 50 rectangular if pulses of $\tau=20 \mu$ s duration each, separated by $5 \mu \mathrm{s}$ was continuously repeated with the repetition rate of $t_{0}=100 \mathrm{~ms}$. 


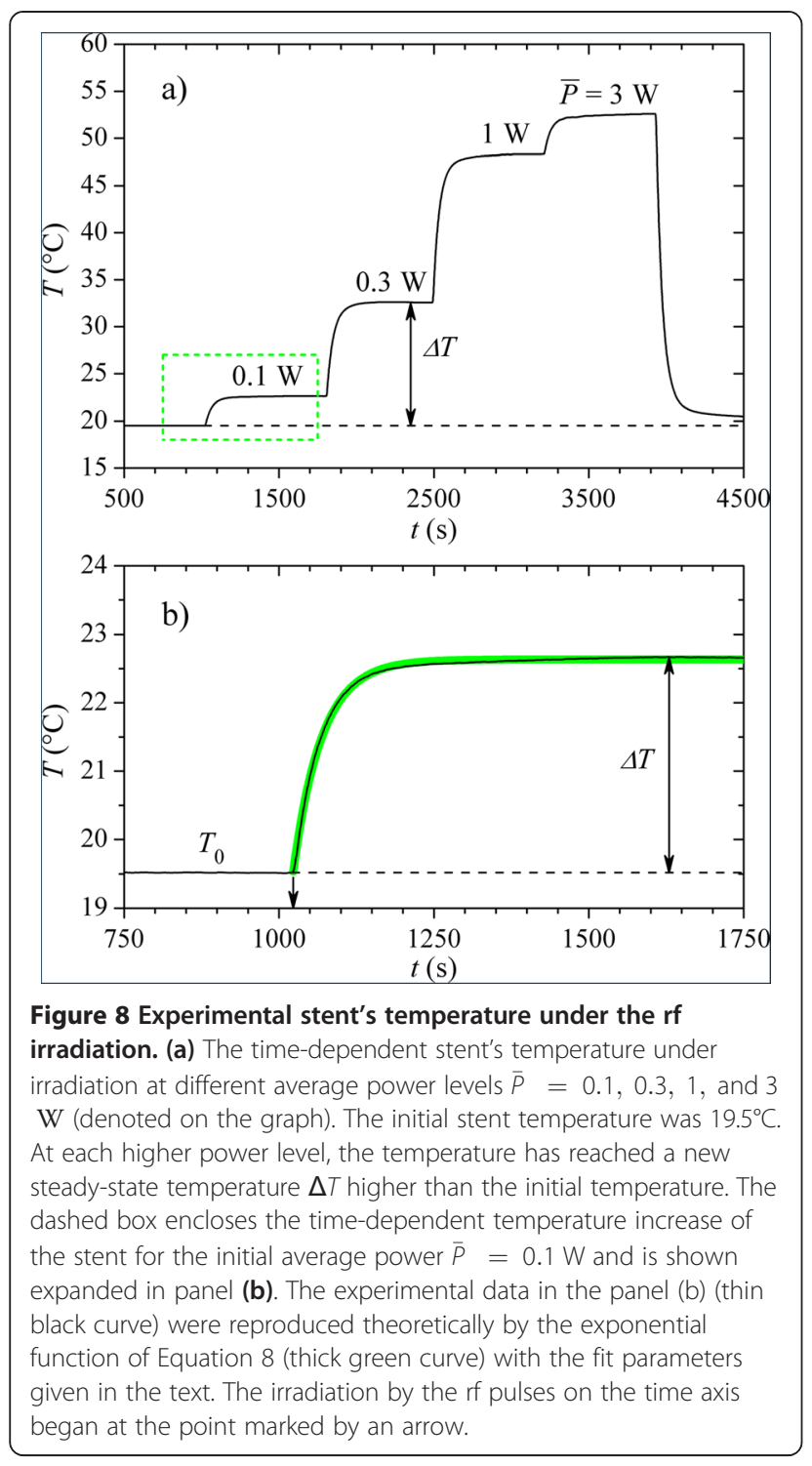

of $52.5^{\circ} \mathrm{C}$ (yielding an increase by as much as $\Delta T=33^{\circ} \mathrm{C}$ from the initial temperature before the irradiation). The values of the steady-state temperature increase $\Delta T$, as a function of $\bar{P}$, are given in Table 1. The detailed shape of the stent's time-dependent temperature $T(t)$ under irradiation by the rf pulses for the initial power level of

Table 1 The temperature increase $\Delta T$ of the stent as a function of the rf power $\bar{P}$

\begin{tabular}{lc}
\hline $\bar{P}(W)$ & $\Delta T\left({ }^{\circ} \mathbf{C}\right)$ \\
\hline 0.1 & 3.1 \\
0.3 & 13.0 \\
1 & 28.8 \\
3 & 33.0 \\
\hline
\end{tabular}

$\bar{P}=0.1 \mathrm{~W}$ (enclosed in a dashed box in Figure 8a), is shown expanded in Figure 8b, where an exponential increase is obvious. We have also checked the increase of the stent's temperature in the air environment at the ambient pressure of $1 \mathrm{bar}$, where the stent's heat supplied by the rf irradiation is taken away more efficiently by the thermal convection to the surrounding air. The highest temperature of the stent in the 1 bar air atmosphere for $\bar{P}=3 \mathrm{~W}$ has dropped to $48^{\circ} \mathrm{C}$, as compared to $52.5^{\circ} \mathrm{C}$ in an identical experiment in the reduced 0.2 bar atmosphere, showing that the thermal convection to the air takes away considerable amount of the heat from the stent.

The above results indicate the possibility of hazardous heating of the stent in the MRI examination by as much as $\Delta T \approx 30^{\circ} \mathrm{C}$. It is, however, important to emphasize that this conclusion is based on experiments with no blood flow through the stent. We shall discuss in the following how does this conclusion change under the blood flow condition.

\section{Comparison between the theory and experiment}

The stent's temperature $T_{s}(t)$ in our rf heating experiment of Figure $8 \mathrm{~b}$ was reproduced theoretically by the Equation 8. Excellent fit (thick green curve in Figure 8b) was obtained using the parameters $\Delta T=3.1^{\circ} \mathrm{C}$ and the time constant $\alpha^{-1}=45 \mathrm{~s}$, confirming that $T_{S}$ reaches the new steady-state value exponentially in time. The experimental $\Delta T$ and $\alpha^{-1}$ values were compared to the theoretical values, calculated from Equations 9 and 7. In the absence of the blood flow, $\Delta T=P_{r f} /[\lambda 2 \pi l / \ln (1+d / R)]$. The role of the vessel wall in the experiment was played by the Teflon jacket of the thickness $d=1 \mathrm{~mm}$ and the thermal conductivity (at $\left.25^{\circ} \mathrm{C}\right) \lambda=0.25 \mathrm{~W} / \mathrm{mK}$ around the stent. Taking the employed $P_{r f}=\bar{P}=0.1 \mathrm{~W}$, and using the stent's geometrical parameters $l=12 \mathrm{~mm}$ and $R=1.25 \mathrm{~mm}$, we obtain $\Delta T=3.1^{\circ} \mathrm{C}$, a value that matches perfectly the experimental one. The parameter $\alpha=[\lambda 2 \pi l / \ln (1+d / R)] / m_{s} c_{s}$ was calculated by using the stent mass $m_{s}=21.7 \mathrm{mg}$ and the specific heat value at $37^{\circ} \mathrm{C} c_{s}=0.46 \mathrm{~J} / \mathrm{gK}$, yielding $\alpha^{-1}=0.3 \mathrm{~s}$. The theoretical time constant $\alpha^{-1}$ is a factor 150 smaller than the experimental one, so that the theory predicts a much faster increase of the stent temperature than observed experimentally. This discrepancy can be understood by noticing that in our experiment, a Pt100 sensor was rigidly attached to the stent and was heated up together with it. The thermal conductivity of the Pt100 ceramic housing is much lower than that of the metallic stent, so that the combined stent-Pt100 system has reached the new steady state temperature in a considerably longer time that it would be reached by the metallic stent alone. 


\section{Application of the model to the coronary stent in the presence of blood flow}

In order to predict the temperature increase of the stainless-steel coronary stent embedded in a coronary artery in vivo in a realistic MRI examination, we calculate the parameter $\Delta T$ for our investigated stent in the presence of the blood flow. We take the geometrical parameters of the as-fabricated stent $l=16 \mathrm{~mm}$ and $R=1.25 \mathrm{~mm}$. For the coronary artery, we take the following order-of-magnitude estimated parameters: the wall thickness $d=1 \mathrm{~mm}$ and the thermal conductivity $\lambda=1 \mathrm{~W} / \mathrm{mK}$ (this value was estimated from the reported thermal conductivity of the human skin plus fat that amounts to $0.73 \mathrm{~W} / \mathrm{mK}$ at $36^{\circ} \mathrm{C}$ and the thermal conductivity of muscles that amounts to $1.91 \mathrm{~W} / \mathrm{mK}$ at $36^{\circ} \mathrm{C}$ (Ducharme and Tikuisis 1991)). For the blood flow through the stent we take a typical volume flow through a coronary artery $\Phi_{b V}=2 \mathrm{ml} / \mathrm{s}$ (Spaan 1991). Since the blood density is $\rho_{b}=1.06 \mathrm{~g} / \mathrm{cm}^{3}$, this yields the blood mass flow $\Phi_{b}=\rho_{b} \Phi_{b V}=2 \mathrm{~g} / \mathrm{s} .2 \mathrm{~g} / \mathrm{s}$. The specific heat of the blood is $c_{b}=3.78 \mathrm{~J} / \mathrm{gK}$. For the stent-to-blood heat transfer efficiency parameter we take an ad hoc value $\varepsilon=0.5 . P_{r f}$ is taken arbitrary as $3 \mathrm{~W}$ (recall that at this $\mathrm{rf}$ power, the stent has heated up by as much as $\Delta T=33^{\circ} \mathrm{C}$ in the absence of the blood flow, as shown in Figure 8a).

Using the above parameter values, the increase of the stent's temperature was calculated from Equation 9 to amount $\Delta T=0.8^{\circ} \mathrm{C}$ only, whereas the blood temperature increase is by $\varepsilon \Delta T=0.4^{\circ} \mathrm{C}$. This $\Delta T$ value is minute as compared to the case where there is no blood flow through the stent. In the absence of the blood flow (setting $\Phi_{b}=0$ in Equation 9), the increase of the stent's temperature would be considerable, $\Delta T=17.6^{\circ} \mathrm{C}$. The reason for the smallness of the $\Delta T$ value in the presence of the blood flow becomes evident by inspecting the denominator of Equation 9 that contains two terms. The first term $\lambda 2 \pi l / \ln (1+d / R)=0.17 \mathrm{~W} / \mathrm{K}$ originates from the heat flow through the vessel wall, whereas the second term ${ }_{\varepsilon} \Phi_{b} c_{b}=3.78 \mathrm{~W} / \mathrm{K}$ originates from the heat taken away by the blood flow. The second term is much larger than the first one, $\varepsilon \Phi_{b} c_{b} /[\lambda 2 \pi l / \ln (1+d / R)]=22$, so that the blood flow efficiently cools the stent in the MRI examination, owing to the much larger heat capacity of the blood, $C_{b}=m_{b} c_{b}$, as compared to the heat capacity of the stent, $C_{s}=m_{s} c_{s}$ (where the ratio of the specific heats is $\left.c_{b} / c_{s}=8.2\right)$. The estimated increase of the stainless-steel coronary stent temperature by $\Delta T$ $=0.8^{\circ} \mathrm{C}$ during MRI in vivo is thus small enough to be considered harmless to the human body.

\section{The problem of restenosis}

The above result of a minute increase of the stent's temperature due to the efficient cooling by the blood flow applies to the normal situation, where the stent's cross section is large enough to enable the rated (unrestricted) blood flow through a coronary vessel, i.e., the stent is fully "open". However, the problem of restenosis is sometimes encountered after the stent insertion in the course of months or years, where the stent becomes partially re-occluded and the flow reduces. Consequently, the reduced flow is no more capable of efficiently cooling the stent in the MRI examination of a patient with a partially re-occluded stent and there may appear a risk of hazardous heating of the stent's surroundings. Defining the blood flow reduction factor $x=\left(1-\Phi_{b} / \Phi_{b}^{0}\right)$ $\times 100$ (in \%) with $\Phi_{b}^{0}$ denoting the unrestricted flow, we are able to predict from Equation 9 the temperature increase of the stent $\Delta T$ for an arbitrary reduced blood flow. The $x=0 \%$ value corresponds to the fully open stent (no flow reduction), whereas $x=100 \%$ corresponds to the fully blocked stent (100\% flow reduction). For the calculation we took the same stent and vessel parameters as before (recall that for these parameters, the model yielded $\Delta T=0.8^{\circ} \mathrm{C}$ for the fully open stent, whereas $\Delta T=17.6^{\circ} \mathrm{C}$ for the fully blocked stent). The graph of the stent's temperature in the body, $T_{s}=37^{\circ} \mathrm{C}$ $+\Delta T$, as a function of the blood flow reduction, is shown in Figure 9. We observe that for the flow reduction between 0 and $90 \%$, the stent's temperature increase is relatively small $\left(T_{\mathrm{s}}\right.$ increases from $37.8^{\circ} \mathrm{C}$ at $0 \%$ reduction to $42.5^{\circ} \mathrm{C}$ at $90 \%$ ), whereas $T_{\mathrm{s}}$ increases drastically for the high flow reduction between $90 \%$ and $100 \%$ (from $42.5^{\circ} \mathrm{C}$ at $90 \%$ to $54.6^{\circ} \mathrm{C}$ at $100 \%$ reduction). This

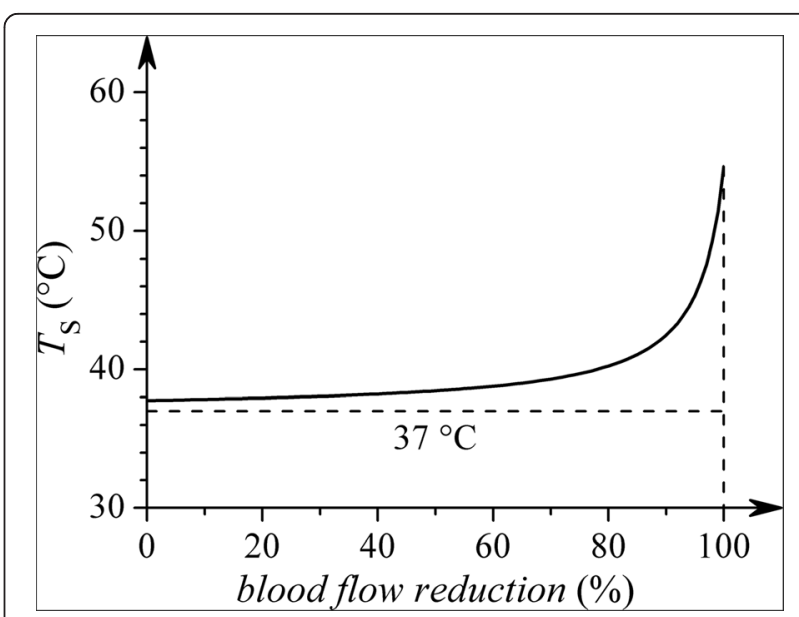

Figure 9 The problem of restenosis in MRI. Theoretical stent's steady-state temperature in the body, $T_{s}=37^{\circ} \mathrm{C}+\Delta T$, under the if irradiation is presented as a function of the blood flow reduction due to restenosis, where $\Delta T$ was calculated from Equation 9 (see text). The blood flow reduction is defined as $x=\left(1-\Phi_{b} / \Phi_{b}^{0}\right) \times 100$ (in \%) with $\Phi_{b}^{0}$ denoting the unrestricted flow. The $x=0 \%$ value corresponds to the fully open stent (no flow reduction), whereas $x=100$ $\%$ corresponds to the fully blocked stent (100\% flow reduction). 
behavior is again a consequence of the much larger heat capacity of the blood as compared to the stent, demonstrating that even a substantially diminished blood flow through the stent is still able to cool it efficiently. In contrast, dangerously high temperatures are developed in the body for the stent occlusion close to $100 \%$.

In the MRI examination, the patient is irradiated by the rf pulses typically for a time of about $20-30$ minutes, which represents the time during which the stent "heater" is switched on. The long heater switch-on time and the high enough temperature for the heat-induced protein coagulation and the formation of blood clots represent a risk of hazardous heating in the stent's surroundings during the MRI of a stented patient with a high stent occlusion between $90 \%$ and $100 \%$. Restenosis thus represents a potentially hazardous heating problem in MRI.

\section{Conclusions}

We have investigated the heating of a metallic coronary stent in a MRI examination. The experimental results presented in this paper are valid for the particular geometrical parameters (length and diameter) of the employed stent, the 316LVM stainless steel material, the proton resonance frequency of $200 \mathrm{MHz}$ (corresponding to the $4.7 \mathrm{~T}$ magnetic field) and the average rf irradiation power up to $3 \mathrm{~W}$. For the highest average rf power of $3 \mathrm{~W}$ employed in our experiments on the stent in the absence of the blood flow through it, the stent has heated up by as much as $\Delta T=33^{\circ} \mathrm{C}$ from the initial temperature before the irradiation. The experimental and theoretical methodologies applied in this study are, however, suitable to investigate other types of stents with different geometries, fabricated of different metallic alloys, e.g., nickel-titanium and cobalt-chromium and other rf irradiation strengths.

The metallic coronary stent acting as a tubular flow heater in the MRI examination was also modeled theoretically, by considering that the stent receives the energy from the $\mathrm{rf}$ electromagnetic field and heats up the surrounding vessel and the blood flowing through it. The analytical model has successfully reproduced the exponential increase of the stent's temperature during our rf heating experiments in the absence of blood flow through the stent. The model was then used to predict the increase of the temperature of the stainless-steel coronary stent embedded in a coronary artery in the presence of blood flow through it, mimicking an in vivo realistic MRI examination. In the normal situation of a fully open (unoccluded) stent, the increase of the stent's temperature as well as the increase of the blood temperature exiting the stent were found minute, of less than $1^{\circ} \mathrm{C}$, so that the blood flow efficiently cools the stent. This is a consequence of the much larger heat capacity of the blood as compared to the heat capacity of the stent. However, should the problem of restenosis occur with time after the stent insertion, where the stent in the vessel becomes partially re-occluded and the flow reduces, there is a risk of hazardous heating. The temperature of the occluded stent may become dangerously high to enable protein coagulation and the formation of blood clots in the stent's surroundings.

\section{Competing interests}

The authors declare that they have no competing interests.

\section{Authors' contributions}

SV carried out the stent heating experiments. MW carried out the physicalproperty measurements of the stent's material. AJ carried out the scanning electron microscopy experiments and the EDS compositional analysis. HJK participated in the application of the theoretical model to the experimental data. JD conceived of the study, developed the analytical model of a stent as a tubular flow heater, and performed the coordination. All authors read and approved the final manuscript.

\section{Author details}

${ }^{1}$ Jožef Stefan Institute, Jamova 39, SI-1000 Ljubljana, Slovenia. ${ }^{2}$ Faculty of Mathematics and Physics, University of Ljubljana, Jadranska 19, SI-1000 Ljubljana, Slovenia. ${ }^{3}$ Institute of Molecular Physics, Polish Academy of Sciences, Smoluchowskiego 17, PL-60-179 Poznań, Poland. ${ }^{4}$ Division of Materials Science, Korea Basic Science Institute, Daejeon 305-333, Republic of Korea.

Received: 5 September 2014 Accepted: 12 November 2014

Published online: 15 January 2015

\section{References}

Ahmed S, Shellock FG (2001) Magnetic resonance imaging safety: implications for cardiovascular patients. J Cardiovasc Magn Reson 3:171-182

ASM International Handbook Committee (1990) Metals handbook, vol 1, 10th edn. ASM International Handbook Committee, Ohio Park

Callaghan PT (1991) Principles of nuclear magnetic resonance microscopy. Clarendon Press, Oxford, p 100

Ducharme MB, Tikuisis P (1991) In vivo thermal conductivity of the human forearm tissues. J Appl Physiol 70:2682-2690

Halliday D, Resnick R, Walker J (2005) Fundamentals of Physics, 7th edn. John Wiley \& Sons, New York, p 493

Hubner PJB (1998) Guide to coronary angioplasty and stenting. Harwood Academic Publishers, Amsterdam

Jost C, Kumar V (1998) Are current cardiovascular stents MRI safe? J Invas Cardiol 10:477-479

Kagetsu ND, Litt AW (1991) Important considerations in measurement of attractive forces on metallic implants in MR imaging. Radiology 179:505-508

Lopič N, Jelen A, Vrtnik S, Jagličić Z, Wencka M, Starc R, Blinc A, Dolinšek J (2013) Quantitative determination of magnetic force on a coronary stent in MRI. J Magn Reson Imaging 37:391-397

Nyenhuis JA, Park SM, Kamondetdacha R, Amjad A, Shellock FG, Rezai A (2005) MRI and implanted medical devices: basic interactions withan emphasis on heating. IEEE Trans Device Mat Rel 5:467-478

Scott NA, Pettigrew RI (1994) Absence of movement of coronary stents after placement in a magnetic resonance imaging field. Am J Cardiol 73:900-901

Shellock FG (2011) Reference manual for magnetic resonance safety, implants and devices. Biomedical Research Publishing Group, Los Angeles, pp 246-251

Shellock FG, Morisoli SM (1994) Ex vivo evaluation of ferromagnetism, heating, and artifacts for heart valve prostheses exposed to a 1.5 Tesla MR system. J Magn Reson Imaging 4:756-758

Shellock FG, Shellock VJ (1999) Metallic stents: evaluation of MR imaging safety. Am J Roentgenol 173:543-547

Spaan JAE (1991) Coronary blood flow: mechanics, distribution and control. Kluwer Academic Publishers, Dordrecht 
Strohm O, Kivelitz D, Gross W, Schulz-Menger J, Liu X, Hamm B, Dietz D, Friedrich MG

(1999) Safety of implantable coronary stents during $1 \mathrm{H}$-magnetic resonance

imaging at 1.0 and 1.5 T. J Cardiovasc Magn Reson 1:239-245

Woods TO (2007) Standards for medical devices in MRI: present and future.

J Magn Reson Imaging 26:1186-1189

Yachia D (1998) Stenting the urinary system. Isis Medical Media, Oxford

Submit your manuscript to a SpringerOpen ${ }^{\circ}$ journal and benefit from:

- Convenient online submission

- Rigorous peer review

- Immediate publication on acceptance

- Open access: articles freely available online

- High visibility within the field

- Retaining the copyright to your article

Submit your next manuscript at $\boldsymbol{\nabla}$ springeropen.com 